\title{
An Urban Scale Inverse Modelling for Retrieving Unknown Elevated Emissions with Building-Resolving Simulations
}

Pramod Kumar ${ }^{\mathrm{a}, *}$, Sarvesh Kumar Singh ${ }^{\mathrm{a}}$, Amir-Ali Feiz ${ }^{\mathrm{a}}$, Pierre Ngae ${ }^{\mathrm{a}}$

${ }^{a}$ LMEE, Université d'Evry Val-d'Essonne, 40 Rue Du Pelvoux 91020 Courcouronnes, France.

\begin{abstract}
This study illustrates an atmospheric source reconstruction methodology for identification of an unknown continuous point release in the geometrically complex urban environments. The methodology is based on the renormalization inversion theory coupled with a building resolving Computational Fluid Dynamics (CFD) modelling approach which estimates the release height along with the projected location on the ground surface and the intensity of an unknown continuous point source in an urban area. An estimation of the release height in a three-dimensional urban environment is relatively more difficult from both technical and computational point of view. Thus, a salient feature of the methodology is to address the problem of vertical structure (i.e. height of a source) in atmospheric source reconstruction in three-dimensional space of an urban region. The inversion methodology presents a way to utilize a CFD model fluidyn-PANACHE in source reconstruction in the urban regions. The described methodology is evaluated with
\end{abstract}

\footnotetext{
*Corresponding author.

Email address: kumar@ufrst.univ-evry.fr (Pramod Kumar)
} 
20 trials of the Mock Urban Field Setting Test (MUST) field experiment in various atmospheric stability conditions varying from neutral to stable and very stable conditions. The retrieved source parameters in all the 20 trials are estimated close to their true source. The source height is retrieved within a factor of two and four in $55 \%$ and $75 \%$ of the MUST trials, respectively. The averaged location error for all 20 trials is obtained $14.54 \mathrm{~m}$ with a minimum of $3.58 \mathrm{~m}$ and maximum of $34.55 \mathrm{~m}$. The averaged estimated release rate for all trials is overpredicted within a factor of 1.48 of the true source intensity and in $85 \%$ of the trials, it is retrieved within in factor of two. In source reconstruction with non-zero measurements, it was observed that the use of all concentration measurements instead of only non-zero essentially makes only the small differences in quality of the source reconstruction and gives a little additional information for better constraining the source parameters. A posteriori uncertainty analysis in the retrieved source parameters is also performed by adding a controlled noise in the concentration measurements and the source reconstruction results were also compared with an earlier study based on the stochastic Bayesian approach for identical 14 MUST trials. The study is useful for emergency regulators to detect an unknown accidental or deliberated continuous point releases in urban regions.

Keywords: CFD, Renormalization, Source reconstruction, MUST field experiment, Urban.

\section{1. Introduction}

$2 \quad$ Fast and accurate identification of an unknown atmospheric tracer source 3 in densely populated and geometrically complex urban environments is es- 
sential to reduce the extent of subsequent exposure and associated mortality caused by an accidental or deliberated incident. An inversion methodology is required to provide information about the locations, height, and emission rates of the unknown sources. A source reconstruction process generally utilizes the ambient concentration measurements of a pollutant observed by a finite number of detectors distributed over a region. However, these concentration measurements, detected over a threshold value of a sensor, alone provide no particular information about the source including its location, height, and release rate.

Atmospheric monitoring in conjunction with source reconstruction modelling has the potential to detect, locate, and quantify the localised emissions into the atmosphere (Luhar et al., 2014). In recent years, the problem of identifying an unknown source on small, medium or large scales is discussed in several studies in the literature (Penenko et al., 2002; Issartel, 2005; Bocquet, 2005; Haupt et al., 2006; Keats et al., 2007; Chow et al., 2008; Sharan et al., 2009; Kovalets et al., 2011; Winiarek et al., 2012; Luhar et al., 2014; Yee et al., 2014; Turbelin et al., 2014; Singh et al., 2015a,b; Kumar et al., 2015b, etc.). These source reconstruction methodologies generally required a numerical dispersion or adjoint model, which prerequisite the realistic windfield in a computational domain. The consideration of a constant flow-field throughout a region is sufficient to compute the adjoint functions in flat and homogeneous terrains, however, it is not as adequate in urban regions. The computation of the realistic flow-fields in urban regions is difficult due to the impacts of local topography, terrain conditions, buildings and other geometrical structures. Buildings in urban regions alter the flow-field, causes updrafts 
and downdrafts, channeling flow between buildings areas of calm winds adjacent to strong winds, etc. (Brown, 2004). However, the building-resolving Computational Fluid Dynamics (CFD) models, that solve the Navier-Stokes fluid dynamics equations using a small grid size (of the order $1 \mathrm{~m}$ or even less) over the complex terrains, can resolve the realistic flow-field in urban regions (Hanna et al., 2004; Kumar et al., 2015a). Accordingly, a CFD model's simulated realistic flow-field in urban regions can be utilize in the dispersion or adjoint models in a source reconstruction process.

Recently, Kumar et al. (2015b) described a source reconstruction methodology for the identification of an unknown continuous point source located at the ground level or at a horizontal plane corresponding to a known or predefined altitude above the ground surface in an urban area. This methodology was based on a recently proposed deterministic renormalization inversion technique (Issartel et al., 2007), coupled with a CFD modelling approach in two-dimensional space in an urban area. However, in this study, the problem of vertical structure (i.e. height of a source) in atmospheric source reconstruction in three-dimensional space of an urban area is not addressed. In reality, an altitude of a release (i.e. source height) is also not known and required to estimate along with the projected release location on the ground surface and the release rate. However, the estimation of the release height in a three-dimensional urban environment is relatively more difficult from both technical and computational point of view. First, the complexity arises due to unresolved vertical structure of the flow and plumes in the urban regions in different atmospheric stability, terrain, and meteorological conditions that affect the feasibility of the source estimation. Second, when the number of 
unknown parameters increases, the computational cost and the complexity of the inversion technique increases. However, the estimation of release height is important along with the projected release location on the ground and intensity, especially when a gas leak or accidental release is elevated or from the rooftops in an urban area.

Thus, the objective of the study reported here is to formulate an inverse atmospheric modelling to estimate the effective release height, along with the projected release location on the ground, and strength of an unknown continuous point source in an urban environment. This study addressed the problem of vertical structure in atmospheric source reconstruction in an urban area. The source reconstruction methodology is based on the renormalization inversion theory combined with a building resolving CFD approach in three-dimensional space in an urban area. In framework of the inversion methodology, a CFD model fluidyn-PANACHE is used for this purpose. This is the first application of the renormalization inversion theory to estimate the height of an unknown continuous point source in an urban environment. The methodology is evaluated with 20 trials of the Mock Urban Setting Test (MUST) field experiment in an urban like environment in various atmospheric stability conditions. The MUST field data is suitable here for the evaluation purpose because the source height and its locations were altered from trial to trial and it can illustrate its applicability to real-world source reconstruction problems in the urban environments. 


\section{Inverse atmospheric modelling for source retrieval}

To estimate the source parameters (i.e. release height, location and intensity), an inversion approach generally uses a finite set of the concentration measurements at some receptors and a source-receptor relationship. This study explores the feasibility of using the renormalization based inversion approach (Issartel, 2005) for three-dimensional (3-D) reconstruction of a continuous atmospheric point source in an urban environment. The adjoint source-receptor relationship is used here and it required an atmospheric dispersion or adjoint model and flow-field in complex urban regions. An adjoint model should properly accounts for the effects of flow inhomogeneities due to the buildings and other obstacles on dispersion in urban regions. The inversion technique is described here for an elevated continuous emission in 3-D discretized space and thus, the time coordinates are ignored in the formulations. However, the technique presented here could be made 4-dimensional to additionally identify the time of an instantaneous point release in the urban regions.

\subsection{Source-receptor relationship}

Assuming that a domain is discretized in $N$ number of grid cells in a 3-dimensional space $\mathbf{x}=(x, y, z)$ and $\mathbf{s} \in \mathbb{R}^{N}$ is an unknown source vector to determine. A finite set of $m$ concentration measurements $\boldsymbol{\mu}=$ $\left(\mu_{1}, \mu_{2}, \ldots, \mu_{m}\right)^{T} \in \mathbb{R}^{m}$ are assumed linear to the emission s. In a receptororiented approach for the source reconstruction that includes solution of an adjoint transport equation (Pudykiewicz, 1998; Hourdin and Talagrand, 2006), the 3-D atmospheric coupling coefficient $a_{i}(\mathbf{x})$ corresponding to each 
receptor formed a source-receptor relationship. The correspondence between the unknown emissions $\mathbf{s}$ and the measurements $\boldsymbol{\mu}$ is defined with the adjoint functions $a_{i}(\mathbf{x})$

$$
\boldsymbol{\mu}=\mathrm{As}+\boldsymbol{\epsilon}
$$

where $\mathbf{A} \in \mathbb{R}^{m \times N}$ is a sensitivity matrix composed of the $m$ time-invariant adjoint functions $a_{i}(\mathbf{x})$ in a 3 -D space and the source vector $\mathbf{s}$ represents discrete components of the unknown emission. The term $\epsilon \in \mathbb{R}^{m}$ represents the total measurement error and it comprises the error due to (i) the detector limitations and (ii) the limited representativity of a dispersion or adjoint model utilized to describe the correspondence between the emissions and the measurements. The volume elements of each grid-cell is incorporated in $\mathbf{s}$, so that it represents the total activity released in a grid-cell. Each column vector of matrix $\mathbf{A}=\left[\mathbf{a}\left(\mathbf{x}_{1}\right), \mathbf{a}\left(\mathbf{x}_{2}\right) \ldots, \mathbf{a}\left(\mathbf{x}_{N}\right)\right]$, where $\mathbf{a}\left(\mathbf{x}_{i}\right) \in \mathbb{R}^{m}$, reflects the potential sensitivity of a grid cell with respect to all $m$ concentration measurements.

For a simple demonstration of the inversion process, a fit to the pollutant concentration $\boldsymbol{\mu}$ at each detector with avail of the sensitivity matrix A can produce a source vector $\mathbf{s}$. However, the inverse problem to estimate $\mathbf{s}$ in Eq. (1) is an ill-posed and highly underdetermined in nature as $m \ll N$. Indeed, in a very general case where the point detectors have a negligible volume compared to the dimensions of the problem, the adjoint functions are singular (with infinite (very large) value) at the position of detectors and have strong concentration gradients (Issartel, 2005; Saide et al., 2011). These peaks are interpreted as artificial information and are regularized by using 
the renormalization assimilation process (Issartel et al., 2007).

\subsection{Renormalized assimilation}

Renormalization inversion theory was originally developed for the estimation of distributed emission sources $\mathbf{s}$ from the concentration measurements (Issartel et al., 2007) and extended to estimate the point source (Sharan et al., 2009). A salient feature of the theory is its distinction of two geometries of the environment where emissions occur. The renormalization inversion theory was further extended to estimate a 2-D ground level point release (or source in a horizontal plane corresponding to a predefined or known vertical plane) in an urban area (Kumar et al., 2015b).

Due to diffusive nature of the transport and strong concentration gradients around the measurement cells, the sensitivity matrix $\mathbf{A}$ is associated with peaks at the sensitivity vectors coinciding with the cells containing measurements (Issartel et al., 2007). These peaks generate artifacts at the point of measurements, that returns unsatisfactory estimates, and need to be regularized. The regularization of peaks in the renormalization source reconstruction process is purely based on the computation of a weight function $\mathbf{W} \in \mathbb{R}^{N \times N}$. The weight matrix $\mathbf{W}$ is purely diagonal in nature with elements $w_{j j}>0$ such that $\sum_{i=1}^{N} w_{j j}=m$. The introduction of $\mathbf{W}$ modifies the sensitivity matrix as $\mathbf{A}_{\mathbf{w}}=\mathbf{A} \mathbf{W}^{-1}$ and thus, the Eq. (1) transforms to :

$$
\boldsymbol{\mu}=\mathbf{A}_{w} \mathbf{W} \mathbf{s}+\boldsymbol{\epsilon}
$$

In order to estimate the unknown source vector $\mathbf{s}$, a constrained optimization problem can be formed to minimize a cost function $J(\mathbf{s})=\mathbf{s}^{T} \mathbf{W} \mathbf{s}$, 
subjected to a constraint $\boldsymbol{\epsilon}=\boldsymbol{\mu}-\mathbf{A}_{w} \mathbf{W s}=0$. The optimization problem can be solved by applying the method of Lagrange multipliers and a unique least-norm estimate $\left(\mathbf{s}_{w}\right)$ of $\mathbf{s}$ can be derived as (Appendix A) :

$$
\mathbf{s}_{w}=\mathbf{A}_{w}^{T} \mathbf{H}_{w}^{-1} \boldsymbol{\mu}
$$

where $\mathbf{H}_{w}^{-1}$ is the inverse of a symmetric positive definite Gram matrix $\mathbf{H}_{w}=$ $\mathbf{A}_{w} \mathbf{W} \mathbf{A}_{w}^{T}$.

The least-norm estimate $\mathbf{s}_{w}$ in Eq. (3) required to determine the appropriate elements $w_{i j}$ of the weight matrix W. Issartel (2005) showed that a unique weight matrix $\mathbf{W}$ exists and satisfies the following conditions :

(i) $\quad w_{j j}>0, \quad($ ii $) \quad \operatorname{trace}(\mathbf{W})=m$, and

(iii) the diagonal terms of $\mathbf{A}_{w}^{T} \mathbf{H}_{w}^{-1} \mathbf{A}_{w}$ are unity, i.e. $\mathbf{a}_{w}^{T}(\mathbf{x}) \mathbf{H}_{w}^{-1} \mathbf{a}_{w}(\mathbf{x}) \equiv 1, \quad$ or equivalently $\operatorname{trace}\left(\mathbf{A}_{w}^{T} \mathbf{H}_{w}^{-1} \mathbf{A}_{w}\right)=N$.

W satisfies an appropriate optimality condition (iii) and this criterion is also called as renormalizing condition (Issartel et al., 2007). An iterative algorithm to compute the weight coefficient $w(\mathbf{x})$ at location $\mathbf{x}$ is described as (Issartel et al., 2007) :

$$
w_{0}(\mathbf{x})=1, \quad \text { and } \quad w_{k+1}(\mathbf{x})=w_{k}(\mathbf{x}) \sqrt{\mathbf{a}_{w k}^{T}(\mathbf{x}) \mathbf{H}_{w k}^{-1} \mathbf{a}_{w k}(\mathbf{x})}
$$

where $k$ is an iteration step. The weights $w(\mathbf{x})$, best removing inversion artifacts, called the renormalizing weights. The renormalized weight function $w(\mathbf{x})$ also referred to as a visibility function, as it is useful in the physical 
interpretation of the extent of the regions seen by the monitoring network (Sharan et al., 2009). The visibility function indicates how well a possible location of a release is observed and, thus, it provides a simple criterion for optimizing the design of the monitoring network.

\subsubsection{Point source retrieval}

A continuous point source is generally characterized by an intensity $q_{0}$ in unit amount of tracer per unit time and a location $\mathbf{x}_{0}=\left(x_{0}, y_{0}, z_{0}\right)$ in a 3 -D space, where $z_{0}$ is the release height and $\left(x_{0}, y_{0}\right)$ is the projected release location on the ground surface. Thus, assuming a priori information about the nature of release, i.e. from a point source, it reduces the number of unknowns to four parameters $\left(q_{0}, x_{0}, y_{0}\right.$, and $\left.z_{0}\right)$ to estimate. A continuous point source can be defined by $s(\mathbf{x})=q_{0} \delta\left(\mathbf{x}-\mathbf{x}_{0}\right)$, where $\delta$ is the Dirac delta function. Substituting this formula of a point source in Eq. (2) gives (Sharan et al., 2009) :

$$
\boldsymbol{\mu}=q_{0} \mathbf{a}_{w}\left(\mathbf{x}_{0}\right) w\left(\mathbf{x}_{0}\right)+\boldsymbol{\epsilon} .
$$

where $\mathbf{a}_{w}\left(\mathbf{x}_{0}\right)=\mathbf{a}\left(\mathbf{x}_{\mathbf{0}}\right) / w\left(\mathbf{x}_{0}\right)$. Thus, $\mathbf{s}_{w}$ from Eq. (3) can be described as :

$$
\mathbf{s}_{w}=q_{0} w\left(\mathbf{x}_{0}\right) \mathbf{A}_{w}^{T} \mathbf{H}_{w}^{-1} \mathbf{a}_{w}\left(\mathbf{x}_{0}\right)
$$

Renormalization condition (iii) from Eq. (4) shows that $\mathbf{s}_{w}$ in Eq. (7) attains its maximum only at a location $\mathbf{x}=\mathbf{x}_{0}$ (because $\mathbf{a}_{w}^{T}(\mathbf{x}) \mathbf{H}_{w}^{-1} \mathbf{a}_{w}\left(\mathbf{x}_{0}\right)=1$ only when $\left.\mathbf{x}=\mathbf{x}_{0}\right)$. Thus, the source location $\left(\mathbf{x}_{0}\right)$ can be estimated on a map by searching the maximum of $\mathbf{s}_{w}$. Once the source location $\mathbf{x}_{0}$ is estimated, its intensity at $\mathbf{x}=\mathbf{x}_{0}$ is determined from Eq. (7) as : 


$$
q_{0}=s_{w}\left(\mathbf{x}_{0}\right) / w\left(\mathbf{x}_{0}\right) .
$$

\subsection{Adjoint functions in urban environments}

Flow-field in urban environments is not homogeneous like generally considered for the flat terrains and it often diverted into unexpected directions by the building and other structures. The unsteady and inhomogeneous flow effect, triggered by the presence of buildings and other geometries, is an influencing factor on the dispersion in urban or industrial regions. Consideration of the constant wind-field throughout a domain to compute the adjoint functions from Gaussian or analytical dispersion models is not appropriate in urban regions (Winiarek, 2014; Kumar et al., 2015a). Compared with simple Gaussian dispersion models or other analytical or empirical approximations, CFD models efficiently predict the obstacles influence on wind patterns and pollutant cloud shapes in complex urban environments (Kumar et al., 2015a). Thus, a CFD model fluidyn-PANACHE is first used to simulate the flow-field in an urban area. This flow-field is then used to compute the adjoint functions.

\subsubsection{Description of the CFD model: fluidyn-PANACHE}

A description of the CFD model fluidyn-PANACHE is given in FluidynPANACHE (2010) and Kumar et al. (2015a). The fluidyn-PANACHE solves the Reynolds Averaged Navier-Stokes (N-S) (RANS) fluid dynamics equations, along with the equations describing conservation of species concentration, mass, heat transfer and energy for a mixture of ideal gases using finite volume numerical techniques. It includes an unsteady RANS CFD solution 
that refers to ensemble-averaging of the N-S equations and resolves only the unsteady mean-flow structures, while it models the turbulence. A built-in automatic 3-D mesh generator is included in the fluidyn-PANACHE that can create the finite-volume mesh around obstacles and body-fitting the terrain undulations.

In fluidyn-PANACHE, ideal gas law is used for the thermodynamic model of mixture of gases. Air is modelled as the moist air with effective properties of the mixture of dry air and water vapour. The Reynolds stresses are modelled using a linear eddy viscosity model (LEVM) (Ferziger and Peric, 2002). Atmospheric turbulence due to shear (flow over ground or over obstacles) as well as due to thermal effects (solar heating of ground, buoyant plumes) is modelled with a two-equations prognostic $k-\epsilon$ model that solves an equation for the turbulent kinetic energy $(k)$ and another equation for its dissipation rate $(\epsilon)$. The atmospheric boundary layer (ABL) processes are built-in the CFD code with different numerical models. The CFD model accounts the stability effects through inflow boundary conditions and includes an ABL model that serves as the interface between the meteorological observations and the boundary conditions. The ABL model is composed of two parts: (i) a micrometeorology model that computes fundamental physical characteristics of the ABL from routine meteorological observations and (ii) a boundary layer model for describing the vertical profiles of wind speed, temperature, and turbulence. Dispersion of gases is modelled by solving the full conservation equations governing the transport of species concentration. The buoyancy model is used to parametrize the body force term in the N-S equations. To couple the pressure and momentum equations in the numerical computa- 
tions, the Semi-Implicit Method for Pressure Linked Equations-Consistent (SIMPLEC or SIMPLE-Consistent) algorithm (Van Doormaal and Raithby, 1984) is utilized.

\subsection{2. $k-\epsilon$ turbulence model}

To resolve the turbulent structure in a computational domain, a modified standard $k-\epsilon$ turbulence model is used. The $k-\epsilon$ model is a two-equation linear eddy viscosity model and describes the mean of a turbulent flow. It solves the transport equations for turbulent kinetic energy, $k$ and its dissipation rate, $\epsilon$. The fluidyn-PANACHE implementation of this model is derived from the standard high-Reynolds number $(R e)$ form with corrections for buoyancy and compressibility (Launder, 2004; Hanjalic, 2005). The $k-\epsilon$ model computes the length and time scales from the local turbulence characteristics. Thus, it can model the turbulent flows subjected to both mechanical shear (obstacles, terrain undulations, canopy) as well as buoyancy (stability and buoyant/heavy gas plumes).

\subsubsection{Boundary conditions}

Recently, Kumar et al. (2015a) discussed the use of proper inflow boundary conditions in a CFD model for dispersion of pollutant in an urban area. The lateral boundaries of the domain are treated as inflow and outflow boundaries based on the direction of the wind with respect to the domain boundary. The top boundary is treated as an outflow boundary and a no-slip bottom boundary condition is defined at the ground surface where the velocity components are set to zero. Standard wall functions (Hanjalic, 2005) are used to compute the drag forces on solid walls in a turbulent boundary layer. 
At the inflow boundary, velocity, temperature, and turbulence variables are specified as follows :

(i) Wind profile : Gryning et al. (2007) wind profile in stable and neutral conditions is used. These profiles are composed of the three different length scales in surface, middle, and upper layers of the ABL, and is applicable in the entire ABL. As the Gryning et al. (2007) wind profile is not suitable for very stable atmospheric conditions, a wind profile based on a similarity function proposed by Beljaars and Holtslag (1991) is used in extreme stability conditions due to its applicability in these stability conditions (Sharan and Kumar, 2010).

(ii) Temperature profile: Monin-Obukhov similarity theory based logarithmic temperature profile is used to describe its vertical variation in neutral and stable conditions.

(iii) Turbulence profiles : The profiles of $k$ and $\epsilon$ based on an approximate analytical solution of one-dimensional $k-\epsilon$ prognostic equation (Yang et al., 2009) are used for inflow boundary conditions. Coefficients in these profiles of $k$ and $\epsilon$ are estimated based on the observations of $k$.

\subsubsection{Adjoint functions}

Adjoint functions are computed in two steps : (i) 3-D converged flow field (u) was computed from the CFD model, and (ii) by reversing the direction of wind by $180^{\circ}$, this wind-field is used to solve the adjoint transport equation (Marchuk, 1995; Pudykiewicz, 1998; Hourdin and Talagrand, 2006):

$$
-\frac{\partial r_{i}}{\partial t}-\mathbf{u} \cdot \nabla r_{i}-\frac{1}{\rho} \nabla \cdot\left(\rho \mathbf{K} \nabla r_{i}\right)=\sigma_{i}
$$


where $r_{i}$ is an adjoint function corresponding to the $i^{\text {th }}$ measurement $\mu_{i}, \sigma_{i}$ is a sampling function associated with $\mu_{i}$ and behave like a source at the receptor locations. The adjoint functions $r_{i}$ are often called as the retroplumes or sensitivity functions (Issartel, 2005). For concentration observations measured by the detector at a point, the geometry of the source at receptor location is generally considered as a 3 -D point source with unit strength in computations of the retroplumes from Eq. (9).

For a continuous release in the atmosphere, adjoint functions become steady with respect to the time. Thus, the time dimension can be discarded and the retroplumes are the function of space variables $\mathbf{x}$ only, i.e. $r_{i}(x, y, z, t)=a_{i}(x, y, z)$.

\section{Mock Urban Setting Test (MUST) field experiment}

The Mock Urban Setting Test (MUST) field experimental was conducted from $6^{\text {th }}$ to $27^{\text {th }}$ September, 2001 at the U.S. Army Dugway Proving Ground (DPG) Horizontal Grid test site (40º $\left.12.606^{\prime} \mathrm{N},-113^{\circ} 10.635^{\prime} \mathrm{W}\right)$ (Biltoft, 2001). In this experiment, an urban like environment was created by placing 120 shipping containers (each $12.2 \mathrm{~m}$ long $\times 2.42 \mathrm{~m}$ wide $\times 2.54 \mathrm{~m}$ high) in 10 rows and 12 columns. These containers formed an approximately $200 \mathrm{~m} \times$ $200 \mathrm{~m}$ region of urban like geometry. Figure 1 shows a layout of the arrangement of the obstacles array in this field experiment. The atmospheric conditions varied from neutral to stable and very stable during the releases in this experiment.

In this study, 20 trials of the MUST experiment are selected for the source reconstruction in various atmospheric conditions. Table 1 presents 
the meteorological, turbulence, and source parameters in each selected trial. The values of the meteorological and turbulence parameters are taken from Yee and Biltoft (2004). In each trial of the MUST experiment, a tracer gas $\left(\mathrm{C}_{3} \mathrm{H}_{6}\right)$ was continuously released for $\approx 15 \mathrm{~min}$ from a point source. The release locations and height were altered slightly from trial to trial, but were always near the first three rows of obstacles (Fig. 1). The tracer gas was released at one of the five vertical heights $0.15,1.3,1.8,2.6$, and $5.2 \mathrm{~m}$ above the ground surface. The concentrations were measured by 40 Digital photoionization detectors (dPIDs) at $1.6 \mathrm{~m}$ above the ground surface, and 8 dPIDs deployed on eight vertical heights at a single location. The 40 detectors at $1.6 \mathrm{~m}$ were arranged in four horizontal sampling lines approximately 25, 60, 95, and $195 \mathrm{~m}$ downwind from the release locations (Yee and Biltoft, 2004). In each trial, $200 \mathrm{~s}$ quasi-steady periods were extracted within each 15 min plume dispersion experiment and the concentration measurements during these periods are used in the estimation of source parameters.

\section{Numerical computations and model application strategy}

The horizontal domain for the calculations considered two computational domains : (i) inner (or nested) domain and (ii) outer domain. The inner domain is defined a size of $250 \mathrm{~m} \times 225 \mathrm{~m}$ (width $\times$ length) that contains the MUST urban geometry of a size $193 \mathrm{~m} \times 171 \mathrm{~m}$. The outer domain is defined approximately four times $(800 \mathrm{~m} \times 800 \mathrm{~m})$ of the size of inner domain. The vertical domains extended from ground level up to $100 \mathrm{~m}$ and $200 \mathrm{~m}$ for inner and outer domain, respectively. These inner and outer domains discretized into 56 and 61 vertical levels, respectively. In both domains, 
lowest 40 vertical levels are set with an uniform $0.25 \mathrm{~m}$ grid spacing that formed the lowest $10 \mathrm{~m}$ of the vertical domains. Above the $10 \mathrm{~m}$ level, the vertical grid spacing are set to uniform $2 \mathrm{~m}$ (5 vertical levels between $10 \mathrm{~m}$ - $20 \mathrm{~m}$ ), $5 \mathrm{~m}$ (4 levels between $20 \mathrm{~m}$ - $40 \mathrm{~m}$ ), $10 \mathrm{~m}$ (6 levels between $40 \mathrm{~m}$ $100 \mathrm{~m}$ ), and, $20 \mathrm{~m}$ (5 levels between $100 \mathrm{~m}$ - $200 \mathrm{~m}$ for outer domain only). 3-D unstructured mesh is generated in both the domains. A grid sensitivity analysis with different mesh resolutions is performed to adapt the mesh for numerical simulation. With refined mesh in the inner domain, near to the obstacles and at locations of the receptors, the 3-D computational domain consists of a total 2849276 grid cells.

The coefficients in approximate analytical profiles of $k$ and $\epsilon$ for inflow boundary conditions are estimated based on the observations of $k$ measured from three horizontal two-dimensional (2-D) sonic anemometer at a $16 \mathrm{~m}$ telescoping pneumatic mast (located $30.5 \mathrm{~m}$ upwind of the front obstacle array). The calculations of the adjoint functions are performed in two steps of the fluidyn-PANACHE. In first step, steady wind-field is computed for each trial of the MUST experiment. In second step, this computed windfield is reversed by $180^{\circ}$ and used in the atmospheric dispersion equation to compute the adjoint function corresponding to each measurement.

The source parameters $\left(q_{0}, x_{0}, y_{0}, z_{0}\right)$ from the renormalization inversion theory coupled with the building resolving CFD modelling approach in each MUST trial are estimated in following steps:

Step 1 : Computation of the flow-field $\mathbf{u}(\mathbf{x})$ in 3-D urban domain using the CFD model fluidyn-PANACHE,

Step 2 : Computation of the adjoint functions $a_{i}(\mathbf{x})$ corresponding to each 
measurement using $180^{\circ}$ reversed flow-field $-\mathbf{u}(\mathbf{x})$ in a dispersion model,

Step 3 : Computation of a matrix $\mathbf{A} \in \mathbb{R}^{m \times N}$ from $m$ adjoint functions $a_{i}(\mathbf{x})$, and Gram matrix $\mathbf{H}=\mathbf{A} \mathbf{A}^{T}$,

Step 4 : Computation of the weight matrix $\mathbf{W} \in \mathbb{R}^{N \times N}$ with an iterative process that generates the matrices $\mathbf{A}_{w}=\mathbf{A} \mathbf{W}^{-1}$ and $\mathbf{H}_{w}=\mathbf{A}_{w} \mathbf{W A}_{w}^{T}$,

Step 5 : Computation of the source vector $\mathbf{s}_{w}=\mathbf{A}_{w}^{T} \mathbf{H}_{w}^{-1} \boldsymbol{\mu}$,

Step 6 : Search for a location $\mathbf{x}_{0}=\left(x_{0}, y_{0}, z_{0}\right)$ corresponds to the maximum of $\mathbf{s}_{w}$ in 3-D domain, and

Step 7 : Calculate the source intensity $q_{0}=s_{w}\left(\mathbf{x}_{0}\right) / w\left(\mathbf{x}_{0}\right)$ at $\mathbf{x}=\mathbf{x}_{0}$.

The weight matrix $\mathbf{W}$ is attained with an accuracy of $O\left(10^{-6}\right)$ within 24 iterations from the iterative algorithm described in section 2.2. The computational methodology for the source reconstruction was programmed in Fortran. After computing the wind-field and adjoint functions from the CFD model, the computational time for retrieval of source parameters in 3-D space for each MUST trial was $\approx 10 \mathrm{~min}$ on a single core of a CentOS release 6.5 machine consisting of Intel(R) Xeon(R) CPU E5-2695 v2 @ 2.40GHz.

Prior to discussion of the source reconstruction results, main assumptions in the inversion methodology formulation are listed as : (i) the number of sources is known a priori, i.e. single source, (ii) the source emissions are steady, i.e. time invariant emission rate, and (iii) nature of the source, i.e. point release. The steady state conditions have been assumed in the present case, which is appropriate given the scale of the MUST field experiment. 
However, this is not likely to be true in real-world urban areas, which are usually of much bigger scale, so the time dependence (of meteorology and dispersion) within the domain would be important in such cases, especially when the averaging times are smaller than the scale of the domain.

\section{Results and discussions}

Source parameters are estimated using (i) synthetic and (ii) real concentration measurements in each trial of the MUST experiment. The synthetic measurements are the modelled concentrations which are used as a first check to test if the inverse model can reproduce the emission used in the derivation of these concentrations. In both types of measurements, source reconstruction are performed for (i) all measurements generated by 40 detectors (including zero and non-zero concentrations) and (ii) only non-zero measurements. Synthetic measurements are free from any instrumental, background, and model errors, and are ideal to validate an inversion methodology for source reconstruction. Knowing the source location $\left(\mathbf{x}_{s}\right)$ and its intensity $q_{s}$ in a 3-D domain, synthetic measurements are essentially computed by $\mu_{i}=q_{s} a_{i}\left(\mathbf{x}_{s}\right)$, where $a_{i}\left(\mathbf{x}_{s}\right)$ is a value of the adjoint function corresponding to the $i^{\text {th }}$ receptor at known source location $\mathbf{x}_{s}$. In each trial of the MUST experiment, source parameters (i.e. $\left.x_{0}, y_{0}, z_{0}, q_{0}\right)$ are exactly estimated with all and non-zero synthetic concentration measurements and this affirms the mathematical consistency of the described source reconstruction methodology in 3-D space of an urban environment.

For real concentration measurements, Figure 2 shows the isopleths of the visibility function $w(\mathbf{x})\left(\mathrm{m}^{-2}\right)$ and normalized source estimate, $s_{w}^{n}(\mathbf{x})=$ 
$s_{w}(\mathbf{x}) / \max \left(\mathbf{s}_{w}\right)$ in the horizontal planes corresponding to the estimated effective source heights $\left(z_{0}\right)$ for five representative trials $3,9,11,15$, and 16 . These trials are corresponding to one trial each from all five true release heights (i.e. source height $\left(z_{s}\right)=0.15 \mathrm{~m}, 1.3 \mathrm{~m}, 1.8 \mathrm{~m}, 2.6 \mathrm{~m}$, and $5.2 \mathrm{~m}$, in trials 3,16 , 11,9 , and 15, respectively) in the MUST field experiment. Also, these trials represent all atmospheric stability conditions (i.e. neutral, stable, and very stable ) observed during the experiment. The isopleths of the weight function and the normalized source estimate for all 20 trials are shown in Supporting Information (SI) Figures S1.1, S1.2, S1.3, \& S1.4. In these Figures (Figures $2, \& \mathrm{~S} 1)$, the contour plots of the source reconstruction results are shown for (i) all measurements, and (ii) only non-zero measurements.

Table 2 presents the source reconstruction results in terms of (i) the error in retrieved source location (location error) $\left(E_{L}\right)$, (ii) the estimated effective release height $\left(z_{0}\right)$, and (iii) the estimated source intensity $\left(q_{0}\right)$. The retrieved source location error $\left(E_{L}\right)$ represents the Euclidean distance of the estimated source location from its true release location. The ratios $\left(q_{0} / q_{s}\right)$ of the estimated $\left(q_{0}\right)$ and the true source release rate $\left(q_{s}\right)$ are also presented for each trial in Table 2. The results in Table 2 are presented for (i) all measurements and (ii) only non-zero measurements. Variation of the number of non-zero concentration measurements and meteorological and atmospheric stability conditions in each MUST trial make the retrieval features different. The isopleths of the renormalized weight function in Figures 2(a) \& S1(a) show the extent of visibility seen by the monitoring network in the horizontal planes corresponding to the estimated effective source heights. It distinguishes the well and poorly seen regions for the source retrieval. The prob- 
ability of a source to be identify is maximum in a well seen region of larger magnitudes of the visibility function and vice versa. It is worth mentioning here that the computations of the visibility function are independent of the concentration measurements. It's computation utilizes only the adjoint functions corresponding to the detectors deployed in a monitoring arrangement and thus, apparently depends on the geometry of a monitoring network. The visibility function attains maximum value at location of the detectors. The distribution of visibility function in the MUST computational domain in Figures 2(a) \& S1(a) exhibits that its magnitude decreases in upwind direction of the monitoring network and vanishes at the most distant regions. Negligible magnitudes of the visibility function in downwind of the monitoring network diminishes the possibility of a sought source in this region.

The use of all concentration measurements instead of only non-zero essentially makes the negligible differences in the quality of the source reconstruction (Table 2, Figures $2 \&$ S1). It suggests that the zero concentrations measurements gave a little additional information for better constraining the source parameters. Irrespective of the varying atmospheric stability conditions in all the trials, the source reconstructions results in 3-D space are well obtained in urban environment of the MUST field experiment.

With real concentration measurements, the contour plots in Figures 2(b) \& S1(b) show the distribution of the normalized source estimate in a horizontal plane corresponding to an altitude of the estimated effective source height $\left(z_{0}\right)$ above the ground surface. The location error in Table 2 and the isopleths of $s_{w}^{n}(\mathbf{x})$ in Figures 2(b) \& S1(b) exhibit that the retrieved source location in each MUST trial is close to the true release location. With all 
concentration measurements, the location error is attained with an average of $14.54 \mathrm{~m}$, with minimum in trial $16\left(E_{L}=3.58 \mathrm{~m}\right)$ and maximum in trial $20\left(E_{L}=34.55 \mathrm{~m}\right)$ (Table 2). The minimum location error is attained in trial $15\left(E_{L}=2.96 \mathrm{~m}\right)$ with an averaged location error of $14.50 \mathrm{~m}$ using only the non-zero concentration measurements (Table 2).

The source intensity $\left(q_{0}\right)$ is retrieved within a factor of two in $\approx 85 \%$ and $\approx 80 \%$ trials of the MUST experiment with all and non-zero measurements, respectively. For all trials, the averaged estimated release rate with all measurements is overpredicted within a factor of 1.48 of the true source intensity, and a comparable overestimation (i.e. the averaged $q_{0} / q_{s}=1.49$ ) is also obtained with only non-zero measurements. In trial 2 , the retrieved source intensity is largely overestimated within an factor of 4.5 of the true release rate (Table 2). A slight overestimation of the intensity from a factor of two (i.e. $\left.q_{0} / q_{s}=2.43\right)$ is obtained in trial 6 . Also, in trial $7, q_{0}$ is slightly underestimated from a factor of two of the true release rate $\left(q_{s}\right)$ with all (i.e. $\left.q_{0} / q_{s}=0.41\right)$ and non-zero measurements (i.e. $\left.q_{0} / q_{s}=0.45\right)$ (Table 2 ).

The tracer gas in all these trials of the MUST field experiment was released at one of the five vertical heights $0.15 \mathrm{~m}, 1.3 \mathrm{~m}, 1.8 \mathrm{~m}, 2.6 \mathrm{~m}$, and 5.2 $\mathrm{m}$ above the ground surface. In $55 \%$ and $75 \%$ of the trials, the source height is estimated respectively within a factor of two and four in 3-D space of MUST urban environment (Table 2). To take the full advantage of different release heights with different projected locations on the ground surface in this field experiment, 3-D source reconstructions results in the trials with each particular release height are discussed separately in the following subsections. 


\subsection{Trials with ground level release at $z_{s}=0.15 \mathrm{~m}$}

In seven trials $1,2,3,6,7,12, \& 13$, the tracer $\mathrm{C}_{3} \mathrm{H}_{6}$ was emitted at 0.15 $\mathrm{m}$ above the ground surface (Table 2) and thus, these trials can be considered corresponding to the ground level releases. These trials were also associated with various atmospheric stability conditions observed during the experiment (neutral: 12, stable: $1,2,6,7, \& 13$, very stable: 3 ) (Table 1). With all concentration measurements, the source height is well retrieved (at $z_{0}=0.125$ $\mathrm{m}$ ) in two trials 7 and 12 in the cells of a horizontal plane corresponds to the lowermost vertical level centred at $0.125 \mathrm{~m}$ of the 3 -dimensional computational domain. The projected source locations correspond to the estimated release height in these trials $7 \& 12$ are also retrieved close to their true positions with the location errors $\left(E_{L}\right) 12.44 \mathrm{~m} \& 5.43 \mathrm{~m}$, respectively. The source intensity in trial 12 is within a factor of two $\left(q_{0} / q_{s}=0.86\right)$; whereas, it is slightly underestimated from a factor of two in trial $7\left(q_{0} / q_{s}=0.41\right)$ (Table 2). With all and non-zero concentration measurements, the averaged locations error in all 7 trials corresponding to the ground level release is 14.02 $\mathrm{m}$ and $13.91 \mathrm{~m}$, respectively. The release rate is estimated within a factor of two in $\approx 57 \%$ of these trials with all and non-zero measurements.

Although, the location and the intensity in trials $1,3,7, \& 13$ are close to the their respective true release parameters, the retrieved source heights were largely overestimated (Table 2). The release heights in 1, 3, 7, \& 13 are estimated as $2.38 \mathrm{~m}, 2.63 \mathrm{~m}, 2.13 \mathrm{~m}, \& 1.38 \mathrm{~m}$, respectively. In trial 2, the estimated location error, $q_{0} / q_{s}$, and height are $30.57 \mathrm{~m}, 4.50$, and $3.38 \mathrm{~m}$, respectively and it gives a large overestimation of the release rate. Irrespective of the inversion methodology, estimating a source height also depends on 
accurately resolving the vertical structure of the computed adjoint functions in 3-D space of an urban geometry. Note that the computation of adjoint functions depends on resolving the vertical structure of the urban boundary layer by a CFD model in different atmospheric stability conditions. The extreme stable conditions in trial 3 may not be resolving properly the vertical structure of the atmospheric boundary layer and thus, influences the estimation of the source height. It is noted that the estimated source parameters in these trials are approximately similar with all and non-zero concentration measurements (Table 2).

\subsection{Trials with source height $z_{s}=1.3 \mathrm{~m}$}

The release height in four trials $16,17,19$, \& 20 was $1.3 \mathrm{~m}$, which is approximately half of the height $(2.54 \mathrm{~m})$ of the MUST urban geometry. In all these trials, source was located outside upwind face of the MUST urban array. In trials 16 and 20, source was positioned $24 \mathrm{~m}$ upwind outside of the MUST array. Whereas, source was located $1 \mathrm{~m}$ upwind of a conex container face in trials 17 and 19. In these trials, it is interesting to test the capability of the source reconstruction methodology to identify a source located outside of the urban environment from the concentration measurements observed from the sensors positioned within an urban region.

With all measurements, the source intensity in these four trials is estimated within a factor of two. The estimated location is also close to the true source position with minimum location error in trial $16\left(E_{L}=3.63 \mathrm{~m}\right)$, maximum in trial $20\left(E_{L}=34.55 \mathrm{~m}\right)$. The averaged location error for these 4 trials is $23.12 \mathrm{~m}$ and $23.31 \mathrm{~m}$ with all and non-zero concentration measurements, respectively. The source heights in trials 19 and 20 are estimated as 
$1.13 \mathrm{~m}$ and $1.38 \mathrm{~m}$, respectively, which are close to the true release height $(1.3$ $\mathrm{m})$. In trials 16 and 17, the release height is estimated as $3.63 \mathrm{~m}$ and $4.38 \mathrm{~m}$, respectively, which are within the factors of 2.8 and 3.4, respectively. With non-zero measurements, the estimated source parameters are approximately similar to that retrieved with all measurements; however, the release height in trial 16 is estimated slightly higher $\left(z_{0}=3.88 \mathrm{~m}\right)$ and lower in trial $20\left(z_{0}\right.$ $=0.13 \mathrm{~m}$ ). Source reconstruction results in these trials exhibit the ability of inversion methodology to retrieve an atmospheric source positioning outside an urban area from the monitoring network within an urban region.

\subsection{Trials with source height $z_{s}=1.8 \mathrm{~m}$}

In five trials $4,5,8,10, \& 11$, the release height was $1.8 \mathrm{~m}$, which is approximately two-thirds of the MUST urban height. In this case, atmospheric stability conditions were observed very stable in trials 4 and 5, stable in 8 and 10, and neutral in trial 11 (Table 1). With all concentration measurements, the source heights in three trials $4,10, \& 11$ are respectively estimated as $2.88 \mathrm{~m}, 1.13 \mathrm{~m}$, and $2.13 \mathrm{~m}$, which are within a factor of two of the true release height $1.8 \mathrm{~m}$ (Table 2). However, in two trials 5 and 8, the estimated effective release heights (i.e. $z_{0}=0.38 \mathrm{~m}$ ) are within a factor of five. In all these trials, the point sources are estimated close to their true positions with an averaged location error of $14.3 \mathrm{~m}$ (with all measurements) and 14.56 (with non-zero measurements) and the intensity is also retrieved within a factor of two (Table 2). It is worth mentioning here also that the estimated source parameters with non-zero concentration measurements are approximately similar to those retrieved with all measurements, except in trial 8 where the retrieved height $\left(z_{0}=0.13 \mathrm{~m}\right)$ is lower than $z_{0}=0.38 \mathrm{~m}$. 


\subsection{Trials with source height $z_{s}=2.6 \mathrm{~m}$}

Rooftop releases at $2.6 \mathrm{~m}$ height above the ground surface were conducted in three trials 9, 14 and 18, where source was positioned at roof of the MUST conex. With all and non-zero measurements, the release height in trial 9 is estimated as $3.63 \mathrm{~m}$ and in trials 14 and 18, the estimated effective source height is $4.13 \mathrm{~m}$, which are within a factor of two of the true source height 2.6 m. Other source parameters in these trials are also estimated close to their true source (Table 2). With all measurements, location errors in trials 9, 14 and 18 are $5.3 \mathrm{~m}, 6.9 \mathrm{~m}$ and $12.33 \mathrm{~m}$, respectively. The retrieved intensities are slightly overestimated and are approximately within a factor of 1.57 of the true release rate. With non-zero concentration measurements, the averaged location error is $7.84 \mathrm{~m}$ which is slightly smaller than $8.18 \mathrm{~m}$ estimated with all measurements. However, the estimated release rates are comparable with all and non-zero measurements in these trials. Source reconstruction results in these trials show the ability of the described inversion methodology to accurately retrieve the rooftop releases in an urban environment.

\subsection{Trial with release at $z_{s}=5.2 \mathrm{~m}$}

In one trial 15 (trial \#2682353), the tracer was released at $5.2 \mathrm{~m}$ height above the ground surface, which makes the source height to be approximately twice of the height $(2.54 \mathrm{~m})$ of the MUST urban geometry. This trial can be considered as an elevated release case for the source reconstruction in stable atmospheric conditions (the Obukhov length $L=120 \mathrm{~m}$ ). The contour plots of the normalized source estimates in a horizontal cross-section plane corresponding to the estimated source height in Figure 2(b) show that the estimated point source in this trial is very close to the true source location 
with all and non-zero concentration measurements (Table 2). With all measurements, the Euclidean distance of the estimated source location from the true source is $4.22 \mathrm{~m}$. The release height is estimated at $4.38 \mathrm{~m}$ above the ground surface which is also close to the true source height $5.2 \mathrm{~m}$ in this trial. The intensity of the estimated point source is retrieved within a factor of two of the true release rate. The retrieved intensity is $306.12 \mathrm{l} / \mathrm{min}$, which is 1.36 times greater than the true release rate $225 \mathrm{l} / \mathrm{min}$ (Table 2).

With non-zero concentration measurements, the source height is estimated same as with all measurements. However, a slightly improvement of the location error $\left(E_{L}=2.96 \mathrm{~m}\right)$ and intensity $\left(q_{0}=285.57 \mathrm{l} / \mathrm{min}, q_{0} / q_{s}=\right.$ 1.27) is observed (Table 2). The source reconstruction results in this trial show that the described methodology is efficient enough to retrieve the source parameters of an elevated release, even a release high enough than the urban height or from roof of a building, in 3-D space of an urban environment.

\subsection{Quantification of posterior uncertainty in estimated source parameters}

Characterization and analysis of the uncertainties in estimated source parameters (i.e. location, height, and intensity) is an important part of a source reconstruction process. In reality, insufficiency and noise in the concentration measurements give rise the uncertainty in estimated source parameters. Other main factors that causes the uncertainty in the retrieved source parameters are the observability of the monitoring network and model representativeness errors. It is noted that, quantification of the posterior uncertainties in the retrieved source parameters is not obvious from the present renormalization inversion methodology. However, these uncertainties can simply be quantified by adding a controlled noise to the concentration measurements 
and compute the source parameters with each noisy measurement. This approach is computationally expensive because it required the estimation of source parameters for several times. However, in renormalization inversion technique, it does not required to compute the weight function with each noisy measurements because the computation of the weight function is independent of the concentration measurements.

To quantify the uncertainties in source parameters in a 3-D space, 50 set of noisy measurements $\left(\mu_{i}^{\text {noisy }}\right.$ ) are generated by adding a controlled $10 \%$ noise in proportion to each concentration measurements $\mu_{i}$, i.e. $\mu_{i}^{\text {noisy }}=\mu_{i}(1+\alpha)$, where $\alpha$ is a Gaussian distributed random number generated in $[-0.1,+0.1]$. For these noisy measurements in each MUST trial, 50 source reconstruction simulations are performed and the average and standard deviation (sd) of these 50 estimated source parameters are calculated.

For all and non-zero measurements with $10 \%$ noise in each trial, the standard deviations (sd) of the estimated location error, height, and intensity are presented in Table 2 and shown by error bar plots in Figures 3(a), (b)\&(c). With all measurements, minimum and maximum standard deviations in retrieved source height is observed in trial $7(0.01 \mathrm{~m})$ and trial $1(7.91 \mathrm{~m})$, respectively (Figure 3(b), Table 2). On an average for all 20 trials, the estimated source height deviates by $0.80 \mathrm{~m}$ from their mean value. However, with non-zero measurements with $10 \%$ noise, the averaged deviation (i.e. sd) of estimated source height for all 20 trials is $1.46 \mathrm{~m}$, with minimum $0.14 \mathrm{~m}$ in trials $4,14, \& 15$ and maximum $8.84 \mathrm{~m}$ in trial 20 (Figure 3(b), Table 2).

With all measurements, the averaged standard deviation in location error $\left(E_{L}\right)$ is $4.87 \mathrm{~m}$ with minimum $0.17 \mathrm{~m}$ in trial 20 and maximum $18.13 \mathrm{~m}$ in 
trial 16 (Figure 3(a), Table 2). On other hand with non-zero measurements, the standard deviation of location error is minimum $0.21 \mathrm{~m}$ in trial 20 and maximum $24.28 \mathrm{~m}$ in trial 18 , with an averaged $5.80 \mathrm{~m}$ of all trials. The release strength with all measurement varies by an averaged of $\approx 18 \%$ from their mean value, with minimum variation in trials 1 and 20, and maximum standard deviation in trial 7 (Figure 3(c), Table 2). Whereas, with nonzero measurements, minimum and maximum standard deviations in retrieved release rate are observed in trial 20 and trial 7, respectively, with an average of $\approx 23 \%$ from their mean value.

It is noted that with non-zero measurements, though the source parameters in a 3-D space are retrieved approximately similar to that estimated by taken all the measurements, the uncertainties in location error, intensity and release height increases slightly. It exhibits that the inclusion of the zero-concentration measurements has importance to reduce the uncertainty in the estimated continuous point source parameters in an urban region.

\subsection{Comparison with Bayesian reconstruction results by Winiarek (2014)}

Recently, Winiarek (2014) performed a source reconstruction study based on a stochastic Bayesian inversion approach to estimate the source parameters in 14 trials of the MUST field experiment. It is worth mentioning here that the present renormalization inversion approach is purely deterministic in nature and does not required any prior information about the point source parameters. However, the successfulness of a stochastic approach, like Bayesian, is dependent on a priori information about the source that generally is not known in reality. In addition, Winiarek (2014) considered only 14 trials; however, this study presented the source reconstruction results in 20 
trials of the MUST field experiment.

With all measurements, the averaged Euclidean distance $\left(E_{L}\right)$ between the retrieved source location and the true source position with present deterministic inversion methodology in these identical 14 trials is $15.5 \mathrm{~m}$, which is smaller than the location error $E_{L}=22.3 \mathrm{~m}$, obtained with the stochastic Bayesian approach by Winiarek (2014). For these 14 trials, the averaged estimated release rate with the present methodology is overpredicted within a factor of 1.5 of the true source intensity which is slightly higher in comparison to the Winiarek (2014) (i.e. the averaged $q_{0} / q_{s}=1.2$ ). In present study, the source intensity in $\approx 93 \%$ (i.e. 13 trials) of these 14 trials is retrieved within a factor of two; whereas, it was within in a factor of two for all the trials in Winiarek (2014). In 50\% of the these trials, the release height is estimated within a factor of two with present study. However, in Winiarek (2014), the estimated release height was within a factor of two in $\approx 43 \%$ of these trials.

It is also noted that the application domain size which Winiarek (2014) considered for the source reconstruction study is only slightly larger than the geometry of MUST array and it is approximately equal to the inner domain size taken in present study. However, in present study, source reconstruction in each trial are applied to approximately four times larger domain than it. Retrieval of a source in a smaller domain can have a higher probability of its accurately estimation in comparison to the retrieval in a larger domain. However, in present inversion methodology, it can be analyzed with the geometrical representation of the visibility function, whether the well and poorly seen regions extend to a larger area up to outer domain in each trial. 


\section{Conclusions}

This study describes an atmospheric source reconstruction methodology based on the renormalization inversion theory coupled with a building resolving CFD modelling approach to locate and quantify a continuous point release in the urban environments. A salient feature of the methodology is to address the problem of vertical structure (i.e. height of a source) in atmospheric source reconstruction in three-dimensional space of an urban area. The inversion methodology is purely deterministic in the sense that it does not required any prior source information and utilizes only a finite number of the ambient concentration measurements and a CFD model to determine the source parameters (i.e. location, height, and strength) in an urban area. The CFD model accounts the influences of buildings and other structures in complex urban regions on flow-field and consequently on adjoint functions in a 3-dimensional space that required in the renormalization inversion technique.

The methodology is evaluated to retrieve the location, height, and strength of a continuous point release in 20 trials of the MUST field experiment in various atmospheric stability conditions varying from neutral to stable and very stable. The retrieved source parameters in all the 20 trials are estimated close to their true source. Different release heights of a point source located at (i) ground level (ii) middle of the buildings (iii) two-thirds of the urban height (iv) roof of the buildings, and (v) above the urban heights, at different horizontal locations in the MUST urban domain are retrieved and analysed. In $55 \%$ and $75 \%$ of the MUST trials, the source height is retrieved within a factor of two and four, respectively. The averaged location error for all 20 trials is attained $14.54 \mathrm{~m}$ with minimum $3.58 \mathrm{~m}$ and maximum $34.55 \mathrm{~m}$. In 
$\approx 85 \%$ of the trials, the source intensity is retrieved within in factor of two. For all trials, the averaged estimated release rate with all measurements is overpredicted within a factor of 1.48 of the true source intensity. The source reconstruction for all the trials is also performed with non-zero measurements only. It was observed that the use of all concentration measurements instead of only non-zero essentially makes the small differences in quality of the source reconstruction and gives a little additional information for better constraining the source parameters.

A posteriori uncertainty analysis in the retrieved source parameters is also carried out with respect to a controlled $10 \%$ noise in the concentration measurements. It is shown that the standard deviation in the release height, location error, and source intensity vary by $0.80 \mathrm{~m}, 4.87 \mathrm{~m}$, and $\approx 18 \%$, respectively from their mean value for all 20 trials. The source reconstruction results are also compared with an earlier study based on a stochastic Bayesian approach for the identical 14 MUST trials, and it was shown that the present methodology is relatively more efficient. Although the urban domain in the present application was less than a kilometer, the methodology can also be used for a larger domain as the CFD models can drive the representative flow-field and dispersion in the larger urban domains. The application of the source reconstruction methodology shows that combining the renormalization inversion theory with a building resolving CFD modelling approach provides an efficient and effective source determination tool in 3-dimensional space of an urban region. 


\section{Appendix A: Minimum weighted-norm solution}

By introducing the Lagrange multipliers $\boldsymbol{\lambda} \in \mathbb{R}^{m}$, an auxiliary function $\mathcal{L}(\mathbf{s}, \boldsymbol{\lambda})$ is formed as:

$$
\mathcal{L}(\mathbf{s}, \boldsymbol{\lambda})=\mathbf{s}^{T} \mathbf{W} \mathbf{s}+\boldsymbol{\lambda}^{T}\left(\boldsymbol{\mu}-\mathbf{A}_{w} \mathbf{W} \mathbf{s}\right)
$$

and solving $\nabla_{\mathbf{s}, \boldsymbol{\lambda}} \mathcal{L}(\mathbf{s}, \boldsymbol{\lambda})=0$, gives:

$$
\begin{aligned}
& \nabla_{\mathbf{s}} \mathcal{L}=2 \mathbf{W} \mathbf{s}-\mathbf{W A}_{w}^{T} \boldsymbol{\lambda}=0 \\
& \nabla_{\boldsymbol{\lambda}} \mathcal{L}=\boldsymbol{\mu}-\mathbf{A}_{w} \mathbf{W} \mathbf{s}=0
\end{aligned}
$$

Eq. (A.2a) implies that $\mathbf{s}=\frac{1}{2} \mathbf{A}_{w}^{T} \boldsymbol{\lambda}$ and substituting it in Eq. (A.2b), one obtains $\boldsymbol{\lambda}=2\left(\mathbf{A}_{w} \mathbf{W A}_{w}^{T}\right)^{-1} \boldsymbol{\mu}=2 \mathbf{H}_{w}^{-1} \boldsymbol{\mu}$. It implies that the source estimate function $\mathbf{s}_{w}=\mathbf{A}_{w}^{T} \mathbf{H}_{w}^{-1} \boldsymbol{\mu}$.

\section{Acknowledgement}

The Mock Urban Setting Test (MUST) database is available at https://mustdpg.dpg.army.mil/. The authors would like to thank the Defense Threat Reduction Agency (DTRA) for providing access to the MUST field experiment dataset. The authors gratefully acknowledge Fluidyn France for use of the CFD model fluidyn-PANACHE. A sincere thanks to Damien Joseph, Dr. Malo Le Guellec and Dr. Claude Souprayen, all from Fluidyn France, for discussions and helping in CFD simulation. The first author, Pramod Kumar, would also like to extend his sincerest thanks and appreciation to Dr. Jean-Pierre Issartel for fruitful discussion of the renormalization inversion theory and his encouragement. This work was supported by the RAPID project DISCARD (no. 132906009) funded by the French Defence Procurement Agency (DGA) and French Ministry of Industry. 


\section{References}

Beljaars, A., Holtslag, A., 1991. Flux parameterization over land surfaces for atmospheric models. Journal of Applied Meteorology 30 (3), 327-341. URL http: //dx .doi .org/10.1175/1520-0450(1991) 030<0327 :FPOLSF>2 . 0 . C0;2

Biltoft, C. A., 2001. Customer report for Mock Urban Setting Test. Tech. rep., West Desert Test Center, U.S. Army Dugway Proving Ground, Dugway, Utah.

Bocquet, M., 2005. Reconstruction of an atmospheric tracer source using the principle of maximum entropy. I: Theory. Quarterly Journal of the Royal Meteorological Society 131 (610), 2191-2208.

URL http://dx.doi.org/10.1256/qj.04.67

Brown, M. J., 2004. Urban dispersion-challenges for fast response modeling. In: Fifth AMS Symposium on the Urban Environment.

Chow, F. K., Kosovic, B., Chan, S., 2008. Source inversion for contaminant plume dispersion in urban environments using building-resolving simulations. Journal of applied meteorology and climatology 47 (6), 1553-1572.

URL http://dx.doi.org/10.1175/2007JAMC1733.1

Ferziger, J. H., Peric, M., 2002. Computational Methods for Fluid Dynamics. Springer Berlin Heidelberg.

Fluidyn-PANACHE, 2010. User Manual. FLUIDYN France / TRANSOFT International, version 4.0.7 Edition. 
Gryning, S.-E., Batchvarova, E., Brmmer, B., Jrgensen, H., Larsen, S., 2007. On the extension of the wind profile over homogeneous terrain beyond the surface boundary layer. Boundary-Layer Meteorology 124 (2), 251-268.

URL http://dx.doi.org/10.1007/s10546-007-9166-9

Hanjalic, K., 2005. Turbulence and transport phenomena: Modelling and simulation. In: Turbulence Modeling and Simulation (TMS) Workshop. Technische Universitt Darmstadt.

Hanna, S. R., Hansen, O. R., Dharmavaram, S., 2004. FLACS CFD air quality model performance evaluation with kit fox, must, prairie grass, and EMU observations. Atmospheric Environment 38 (28), 4675-4687.

URL http://www.sciencedirect.com/science/article/pii/S1352231004005345

Haupt, S. E., Young, G. S., Allen, C. T., 2006. Validation of a receptordispersion model coupled with a genetic algorithm using synthetic data. Journal of applied meteorology and climatology 45 (3), 476-490.

URL http://dx.doi.org/10.1175/JAM2359.1

Hourdin, F., Talagrand, O., 2006. Eulerian backtracking of atmospheric tracers. I: Adjoint derivation and parametrization of subgrid-scale transport. Quarterly Journal of the Royal Meteorological Society 132 (615), 567-583. URL http://dx.doi.org/10.1256/qj.03.198.A

Issartel, J.-P., 2005. Emergence of a tracer source from air concentration measurements, a new strategy for linear assimilation. Atmospheric Chemistry and Physics 5 (1), 249-273.

URL http://www . atmos-chem-phys . net/5/249/2005/ 
Issartel, J.-P., Sharan, M., Modani, M., 2007. An inversion technique to retrieve the source of a tracer with an application to synthetic satellite measurements. Proceedings of the Royal Society of London A: Mathematical, Physical and Engineering Sciences 463 (2087), 2863-2886.

URL http: //rspa.royalsocietypublishing.org/content/463/2087/2863. abstract

Keats, A., Yee, E., Lien, F.-S., 2007. Bayesian inference for source determination with applications to a complex urban environment. Atmospheric Environment 41 (3), $465-479$.

URL http://www.sciencedirect.com/science/article/pii/S1352231006008703

Kovalets, I. V., Andronopoulos, S., Venetsanos, A. G., Bartzis, J. G., 2011. Identification of strength and location of stationary point source of atmospheric pollutant in urban conditions using computational fluid dynamics model. Mathematics and Computers in Simulation 82 (2), 244 $-257$.

URL http://www.sciencedirect.com/science/article/pii/S0378475411001686

Kumar, P., Feiz, A.-A., Ngae, P., Singh, S. K., Issartel, J.-P., 2015a. CFD simulation of short-range plume dispersion from a point release in an urban like environment. Atmospheric Environment 122, 645 - 656 .

URL http://www.sciencedirect.com/science/article/pii/S1352231015304465

Kumar, P., Feiz, A.-A., Singh, S. K., Ngae, P., Turbelin, G., 2015b. Reconstruction of an atmospheric tracer source in an urban-like environment. Journal of Geophysical Research: Atmospheres 120 (24), 12589-12604, 2015JD024110.

URL http://dx.doi .org/10.1002/2015JD024110 
Launder, B., 2004. Turbulence modelling of buoyancy-affected flows. In: Singapore Turbulence Colloquium.

Luhar, A. K., Etheridge, D. M., Leuning, R., Loh, Z. M., Jenkins, C. R., Yee, E., 2014. Locating and quantifying greenhouse gas emissions at a geological CO2 storage site using atmospheric modeling and measurements. Journal of Geophysical Research: Atmospheres 119 (18), 10,959-10,979, 2014JD021880.

URL http://dx.doi.org/10.1002/2014JD021880

Marchuk, G. I., 1995. Adjoint Equations and Analysis of Complex Systems. Springer Netherlands.

Penenko, V., Baklanov, A., Tsvetova, E., 2002. Methods of sensitivity theory and inverse modeling for estimation of source parameters. Future Generation Computer Systems 18 (5), 661 - 671, \{ICCS2001\}. URL http://www.sciencedirect.com/science/article/pii/S0167739X02000316

Pudykiewicz, J. A., 1998. Application of adjoint tracer transport equations for evaluating source parameters. Atmospheric Environment 32 (17), 3039-3050.

URL http://www.sciencedirect.com/science/article/pii/S1352231097004809

Saide, P., Bocquet, M., Osses, A., Gallardo, L., 2011. Constraining surface emissions of air pollutants using inverse modelling: method intercomparison and a new two-step two-scale regularization approach. Tellus B 63 (3), $360-370$.

URL http://dx.doi.org/10.1111/j.1600-0889.2011.00529.x 
Sharan, M., Issartel, J.-P., Singh, S. K., Kumar, P., 2009. An inversion technique for the retrieval of single-point emissions from atmospheric concentration measurements. Proceedings of the Royal Society of London A: Mathematical, Physical and Engineering Sciences 465, 2069-2088. URL http://rspa.royalsocietypublishing.org/content/early/2009/04/09/rspa. 2008.0

Sharan, M., Kumar, P., 2010. Estimation of upper bounds for the applicability of non-linear similarity functions for non-dimensional wind and temperature profiles in the surface layer in very stable conditions. Proceedings of the Royal Society of London A: Mathematical, Physical and Engineering Sciences 467 (2126), 473-494.

Singh, S. K., Sharan, M., Singh, A. K., 2015a. Reconstructing height of an unknown point release using least-squares data assimilation. Quarterly Journal of the Royal Meteorological Society 141 (689), 1376-1388.

URL http://dx.doi.org/10.1002/qj.2446

Singh, S. K., Turbelin, G., Issartel, J.-P., Kumar, P., Feiz, A. A., 2015b. Reconstruction of an atmospheric tracer source in fusion field trials: Analyzing resolution features. Journal of Geophysical Research: Atmospheres 120 (12), 6192-6206, 2015JD023099.

URL http://dx.doi.org/10.1002/2015JD023099

Turbelin, G., Singh, S. K., Issartel, J.-P., 2014. Reconstructing source terms from atmospheric concentration measurements: Optimality analysis of an inversion technique. Journal of Advances in Modeling Earth Systems 6 (4), $1244-1255$.

URL http://dx.doi.org/10.1002/2014MS000385 
Van Doormaal, J. P., Raithby, G. D., 1984. Enhancements of the simple method for predicting incompressible fluid flows. Numerical Heat Transfer 7 (2), 147-163.

URL http://dx.doi.org/10.1080/01495728408961817

Winiarek, V., 2014. Dispersion atmosphérique et modélisation inverse pour la reconstruction de sources accidentelles de polluants. Ph.D. thesis, Université Paris-Est.

URL https://pastel.archives-ouvertes.fr/tel-01004505

Winiarek, V., Bocquet, M., Saunier, O., Mathieu, A., 2012. Estimation of errors in the inverse modeling of accidental release of atmospheric pollutant: Application to the reconstruction of the cesium-137 and iodine-131 source terms from the fukushima daiichi power plant. Journal of Geophysical Research: Atmospheres 117 (D5), D05122, d05122.

URL http://dx.doi.org/10.1029/2011JD016932

Yang, Y., Gu, M., Chen, S., Jin, X., 2009. New inflow boundary conditions for modelling the neutral equilibrium atmospheric boundary layer in computational wind engineering. Journal of Wind Engineering and Industrial Aerodynamics 97 (2), 88-95.

URL http://www.sciencedirect.com/science/article/pii/S0167610508001815

Yee, E., Biltoft, C., 2004. Concentration fluctuation measurements in a plume dispersing through a regular array of obstacles. Boundary-Layer Meteorology $111(3), 363-415$.

URL http://dx.doi.org/10.1023/B\%3ABOUN.0000016496.83909.ee 
Yee, E., Hoffman, I., Ungar, K., 2014. Bayesian inference for source reconstruction: A real-world application. International Scholarly Research Notices 2014, 1-12.

URL http://dx.doi .org/10.1155/2014/507634

\section{List of Tables}

1 The selected 20 trials of the MUST field experiments with source, meteorological, and turbulence parameters (Biltoft, 2001; Yee and Biltoft, 2004). $z_{s}, t_{s}$, and $q_{s}$ are respectively the effective source height, duration, and release rate. $S_{04}$ and $\alpha_{04}$ are the wind speed and direction respectively at a 4 m level of mast $\mathrm{S}$. The Obukhov length $(L)$, friction velocity $\left(u_{*}\right)$, and turbulent kinetic energy $(k)$ are at $4 \mathrm{~m}$ level of tower T. The Trial No. 1-20 are assigned here for just continuation and simplicity and these do not correspond to the same trial no. as assigned for a Trial name in the MUST experiment. . . 42

2 Source reconstruction results for all 20 trials of MUST field experiment by considering all (i.e. $m=40$ ) and non-zero measurements in each trial. Here, $m$ is the no. of measurements considered for source retrieval in each trial. $q_{s}$ and $q_{0}$ represent the true and estimated release rates, respectively. $z_{s}$ and $z_{0}$ are respectively the true and estimated source height. The location error $\left(E_{L}\right)$ represents the Euclidian distance of the retrieved source location from the true position. $E_{q}=q_{0} / q_{s}$ is a ratio of the retrieved and the true release rates in each trial. 43 


\section{List of Figures}

1 Layout of the MUST urban geometry showing 120 containers, source, and receptor locations. The black circles denote the position of receptors. The stars denote the source locations only one was operational in a given trial. . . . . . . . . . . . . 44

2 Isopleths of the weight functions $w(\mathbf{x})\left(m^{-2}\right)$ (in panel a) and the normalized source estimate $s_{w}^{n}(\mathbf{x})=s_{w}(\mathbf{x}) / \max \left(\mathbf{s}_{w}\right)$ (in panel b) in a horizontal plane corresponding to the estimated effective source height $\left(z_{0}\right)$ in trials $3,9,11,15$, and 16 , with all and non-zero concentration measurements. The black and white filled circles in panels (b) show the true and estimated source locations, respectively. . . . . . . . . . . . . . . 45

3 Bar plots for (a) Location error $\left(E_{L}\right)$, (b) estimated effective source height $\left(z_{0}\right)$, and (c) source intensity ratios $\left(E_{q}=q_{0} / q_{s}\right)$. The corresponding uncertainties (standard deviation) are represented by the errorbars. . . . . . . . . . . . . . . 46 
Table 1: The selected 20 trials of the MUST field experiments with source, meteorological, and turbulence parameters (Biltoft, 2001; Yee and Biltoft, 2004). $z_{s}, t_{s}$, and $q_{s}$ are respectively the effective source height, duration, and release rate. $S_{04}$ and $\alpha_{04}$ are the wind speed and direction respectively at a $4 \mathrm{~m}$ level of mast $\mathrm{S}$. The Obukhov length $(L)$, friction velocity $\left(u_{*}\right)$, and turbulent kinetic energy $(k)$ are at $4 \mathrm{~m}$ level of tower T. The Trial No. 1-20 are assigned here for just continuation and simplicity and these do not correspond to the same trial no. as assigned for a Trial name in the MUST experiment.

\begin{tabular}{|c|c|c|c|c|c|c|c|c|c|}
\hline $\begin{array}{l}\text { Trial } \\
\text { No. }\end{array}$ & $\begin{array}{l}\text { Trial Name } \\
\text { (JJJhhmm) }\end{array}$ & $\begin{array}{c}q_{s} \\
(1 / \min )\end{array}$ & $\begin{array}{c}t_{s} \\
(\min )\end{array}$ & $\begin{array}{c}z_{s} \\
(\mathrm{~m})\end{array}$ & $\begin{array}{c}S_{04} \\
(\mathrm{~m} / \mathrm{s})\end{array}$ & $\begin{array}{c}\alpha_{04} \\
(\operatorname{deg})\end{array}$ & $\begin{array}{c}u_{*} \\
(\mathrm{~m} / \mathrm{s})\end{array}$ & $\begin{array}{c}L \\
(\mathrm{~m})\end{array}$ & $\begin{array}{c}k \\
\left(m^{2} s^{-2}\right)\end{array}$ \\
\hline 1 & 2640138 & 175 & 21 & 0.15 & 2.35 & 17 & 0.26 & 91 & 0.359 \\
\hline 2 & 2640246 & 200 & 15 & 0.15 & 2.01 & 30 & 0.25 & 62 & 0.306 \\
\hline 3 & 2671852 & 200 & 22 & 0.15 & 3.06 & -49 & 0.32 & 330 & 0.436 \\
\hline 4 & 2671934 & 200 & 15 & 1.8 & 1.63 & -48 & 0.08 & 5.8 & 0.148 \\
\hline 5 & 2672033 & 200 & 15 & 1.8 & 2.69 & -26 & 0.17 & 4.8 & 0.251 \\
\hline 6 & 2672101 & 200 & 14 & 0.15 & 1.89 & -10 & 0.16 & 7.7 & 0.218 \\
\hline 7 & 2672150 & 200 & 16 & 0.15 & 2.30 & 36 & 0.35 & 150 & 0.409 \\
\hline 8 & 2672213 & 200 & 15 & 1.8 & 2.68 & 30 & 0.35 & 150 & 0.428 \\
\hline 9 & 2672235 & 200 & 15 & 2.6 & 2.32 & 36 & 0.26 & 48 & 0.387 \\
\hline 10 & 2672303 & 200 & 19 & 1.8 & 2.56 & 17 & 0.25 & 74 & 0.367 \\
\hline 11 & 2681829 & 225 & 15 & 1.8 & 7.93 & -41 & 1.10 & 28000 & 1.46 \\
\hline 12 & 2681849 & 225 & 16 & 0.15 & 7.26 & -50 & 0.76 & 2500 & 0.877 \\
\hline 13 & 2682256 & 225 & 15 & 0.15 & 5.02 & -42 & 0.66 & 240 & 0.877 \\
\hline 14 & 2682320 & 225 & 15 & 2.6 & 4.55 & -39 & 0.50 & 170 & 0.718 \\
\hline 15 & 2682353 & 225 & 15 & 5.2 & 4.49 & -47 & 0.44 & 120 & 0.727 \\
\hline 16 & 2692054 & 225 & 22 & 1.3 & 3.34 & 39 & 0.36 & 170 & 0.362 \\
\hline 17 & 2692131 & 225 & $1_{42}$ & 1.3 & 4.00 & 39 & 0.42 & 220 & 0.582 \\
\hline 18 & 2692157 & 225 & $15^{42}$ & 2.6 & 2.98 & 43 & 0.39 & 130 & 0.505 \\
\hline 19 & 2692223 & 225 & 15 & 1.3 & 2.63 & 26 & 0.35 & 120 & 0.484 \\
\hline 20 & 2692250 & 225 & 17 & 1.3 & 3.38 & 36 & 0.37 & 130 & 0.537 \\
\hline
\end{tabular}




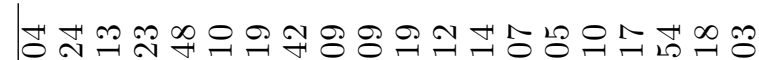

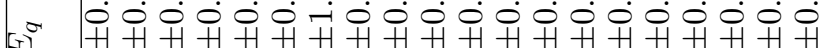

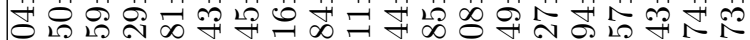
ヘ่

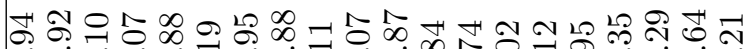

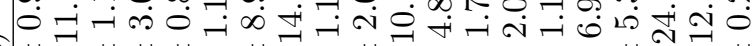

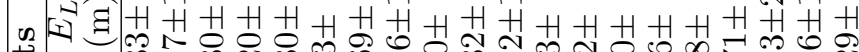
ป

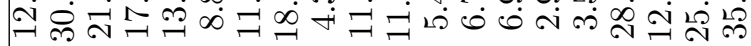

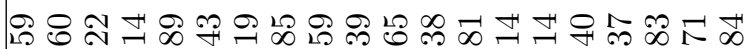

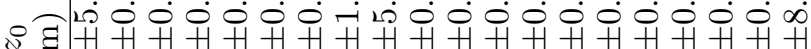

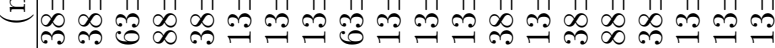

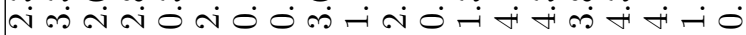
๑ั

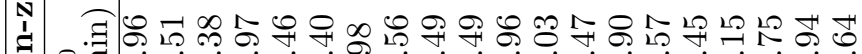

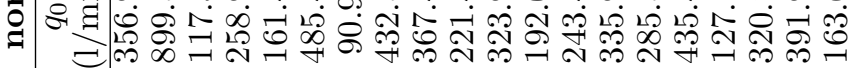

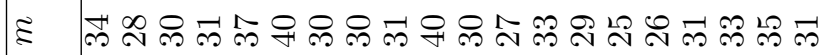

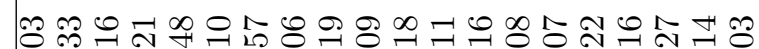

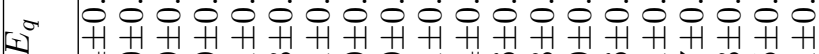

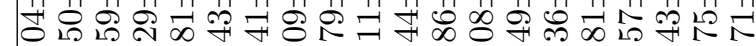
ヘ่

ஜ유서 0 -

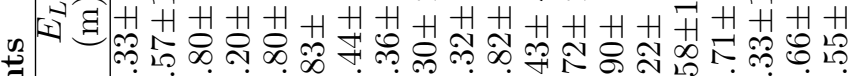
包

పా 。

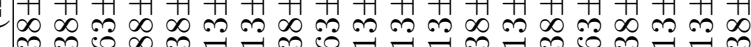

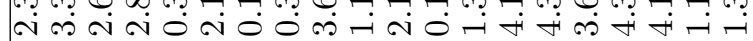
业

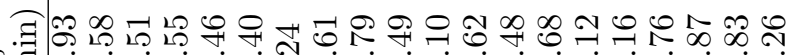
\&

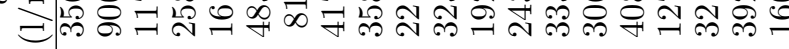

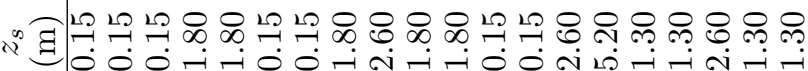
寻官客客客客客客客客客 ๙ छ्छิ ๘ี Z

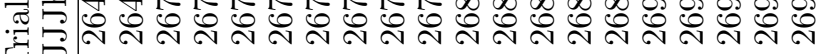
㞼官け 


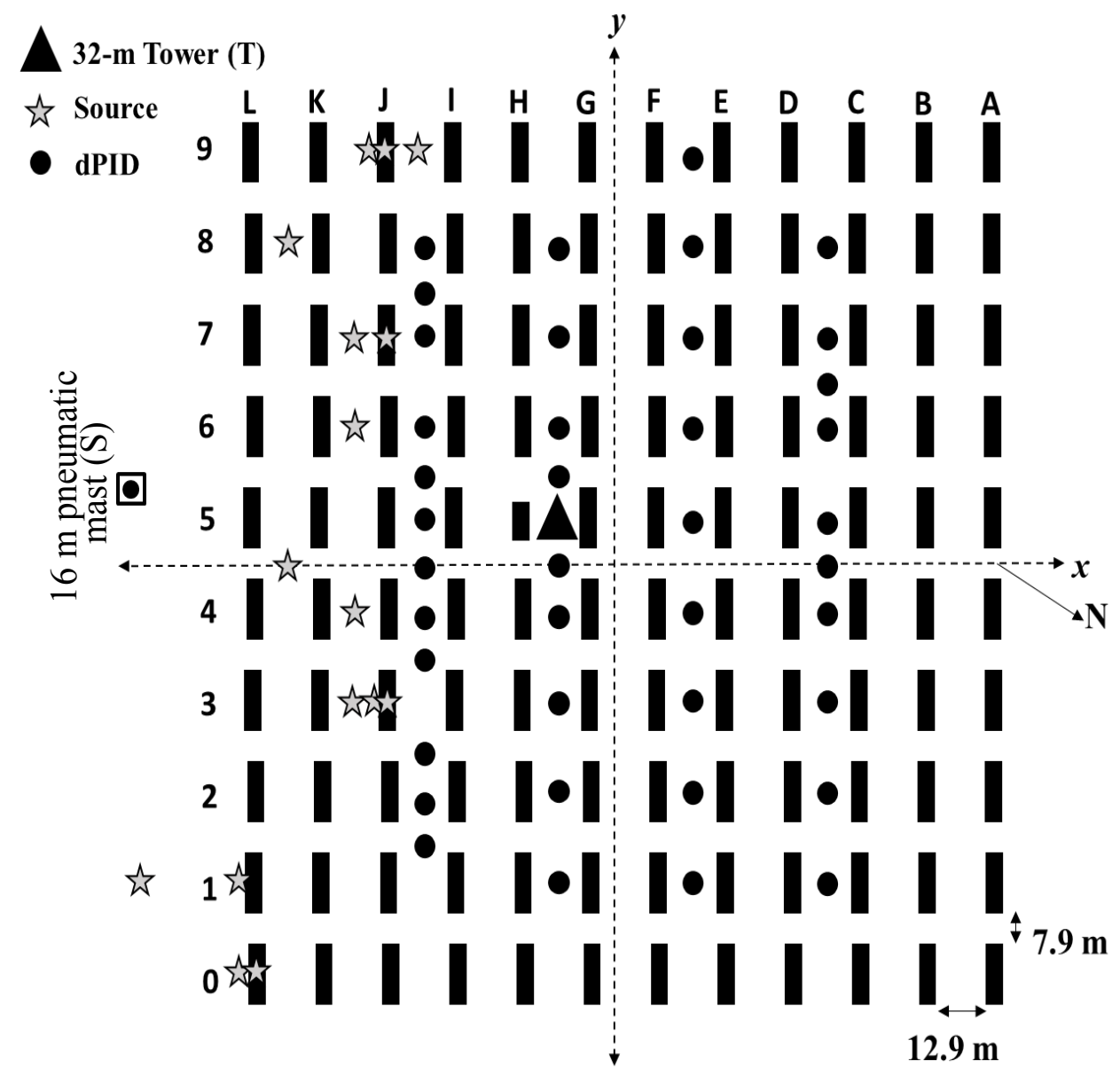

Figure 1: Layout of the MUST urban geometry showing 120 containers, source, and receptor locations. The black circles denote the position of receptors. The stars denote the source locations - only one was operational in a given trial. 
all measurements

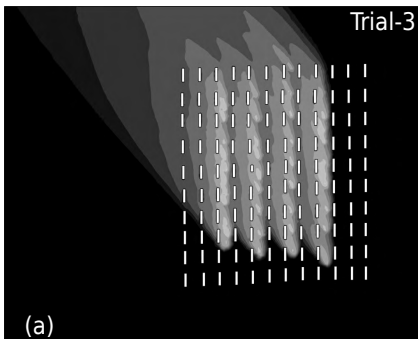

(a)

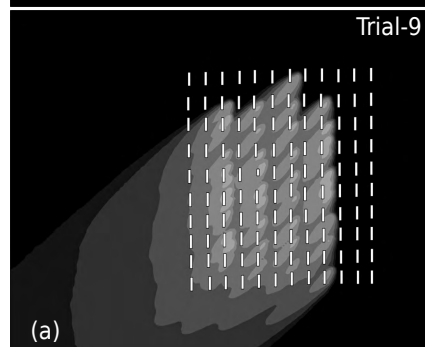

(b)

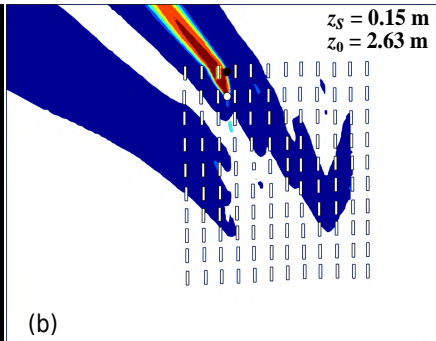

$z_{S}=2.60 \mathrm{~m}$
$z_{0}=3.63 \mathrm{~m}$

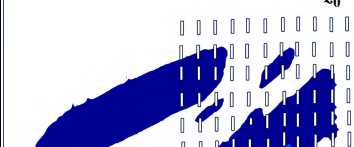

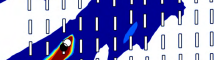

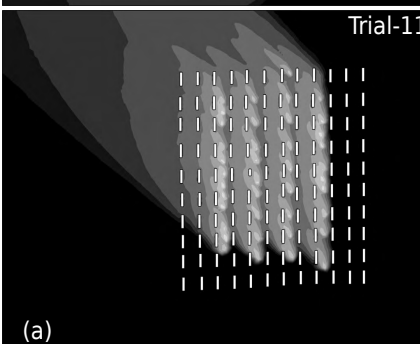

(a)

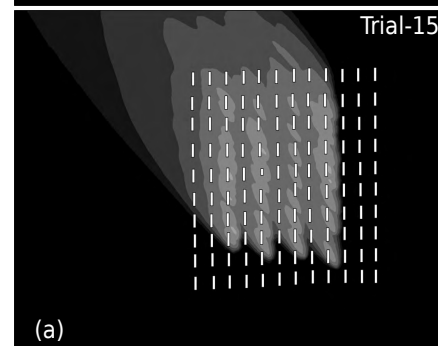

(a)

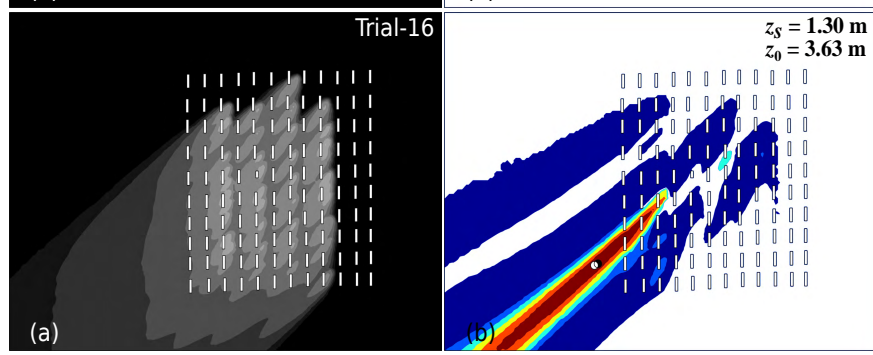

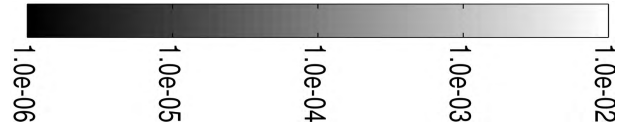

(b)

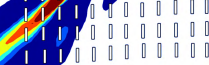

non-zero measurements

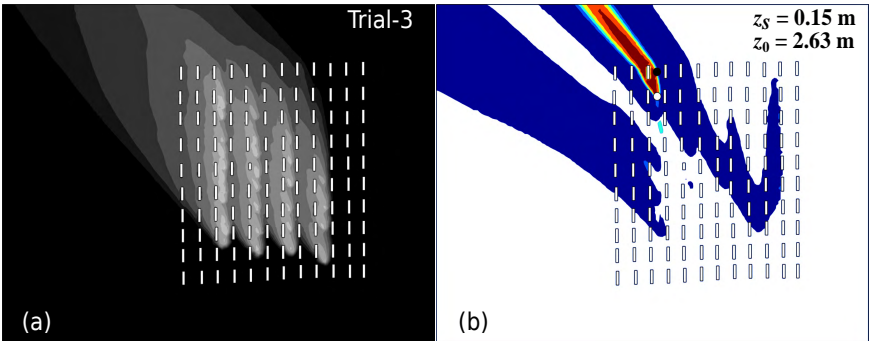

(a)
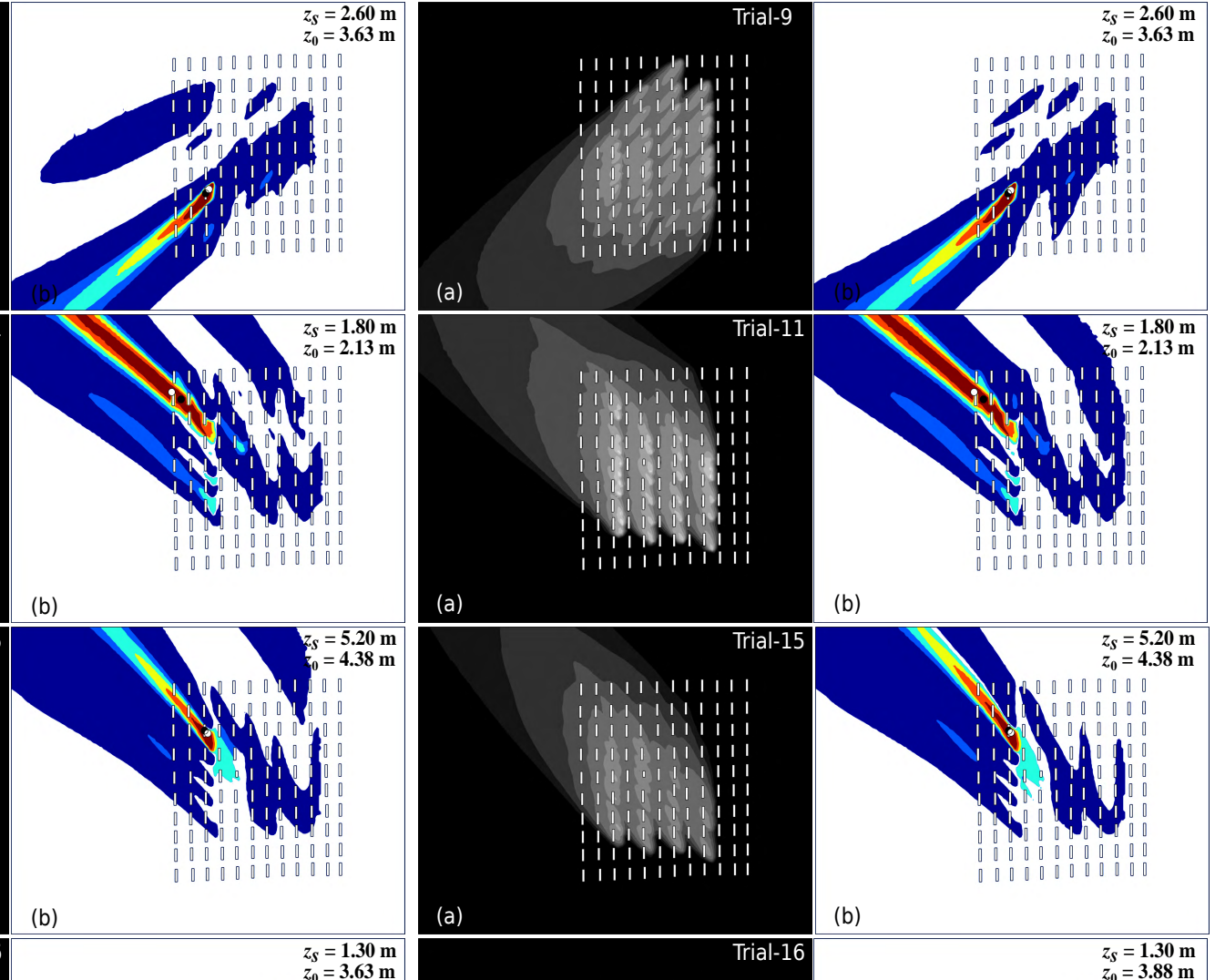

(b)

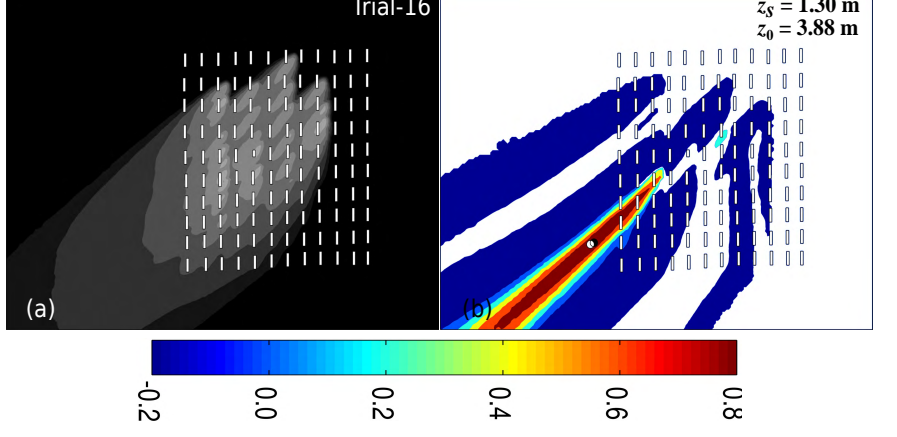

Figure 2: Isopleths of the weight functions $w(\mathbf{x})\left(\mathrm{m}^{-2}\right)$ (in panel a) and the normalized source estimate $s_{w}^{n}(\mathbf{x})=s_{w}(\mathbf{x}) / \max \left(\mathbf{s}_{w}\right)$ (in panel b) in a horizontal plane corresponding to the estimated effective source height $\left(z_{0}\right)$ in trials $3,9,11,15$, and 16 , with all and non-zero concentration measurements. The black and white filled circles in panels (b) show the true and estimated source locations, respectively. 

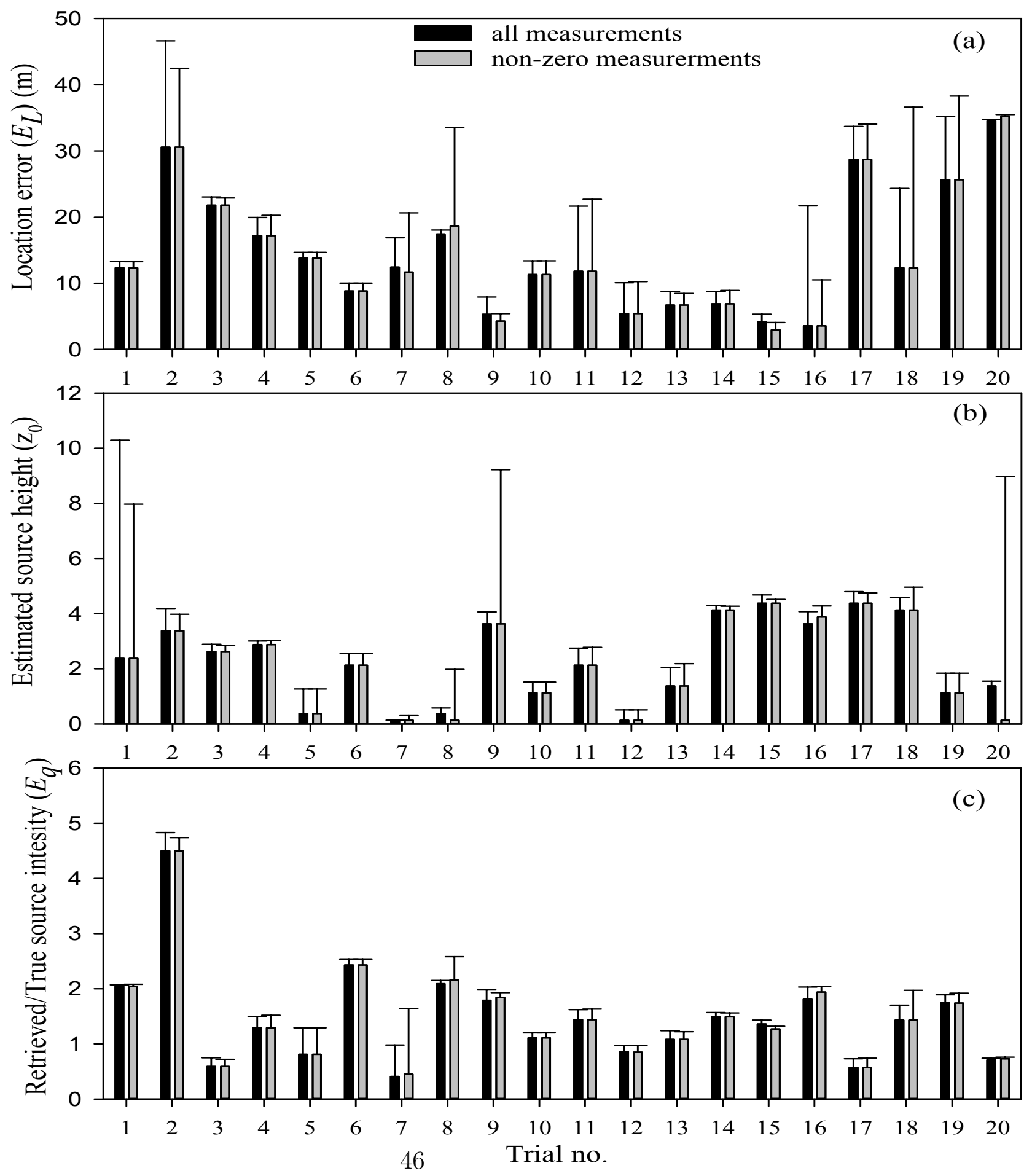

Figure 3: Bar plots for (a) Location error $\left(E_{L}\right)$, (b) estimated effective source height $\left(z_{0}\right)$, and (c) source intensity ratios $\left(E_{q}=q_{0} / q_{s}\right)$. The corresponding uncertainties (standard deviation) are represented by the errorbars. 